\title{
Rough Fibrils Provide a Toughening Mechanism in Biological Fibers
}

\author{
Cameron P. Brown, ${ }^{+, \neq, \S, \perp, *}$ Catalin Harnagea, ${ }^{\S}$ Harinderjit S. Gill, ${ }^{\dagger}$ Andrew J. Price, ${ }^{\dagger}$ Enrico Traversa, ${ }^{\ddagger, \perp, l l}$ \\ Silvia Licoccia, ${ }^{\neq, \perp}$ and Federico Rosei ${ }^{\S, \perp, *}$ \\ ${ }^{\dagger}$ Botnar Research Centre, Nuffield Department of Orthopaedics, Rheumatology and Musculoskeletal Sciences, University of Oxford, Oxford, U.K., ${ }^{\ddagger}$ Centro NAST, \\ Dipartimento di Scienze e Tecnologie Chimiche, Università di Roma Tor Vergata, Roma, Italy, Institut National de la Recherche Scientifique, Énergie, Matériaux et \\ Télécommunications, Université du Québec, Canada, ${ }^{\perp}$ Italy-Quebec Joint Laboratory in Nanostructured Materials for Energy, Catalysis and Biomedical Applications, \\ and "International Research Center for Materials Nanoarchitectonics, National Institute for Materials Science, Tsukuba, Japan
}

$\mathrm{T}$ he toughness of certain biological materials results from the balance of properties in 'hierarchical structures' or over a range of length scales. ${ }^{1,2}$ At the $1-10 \mathrm{~nm}$ scale in spider silk (see Figure 1), a combination of high strength and high toughness can be achieved by the coupling of crystalline with amorphous regions and manipulating the size of crystals, ${ }^{3-5}$ sacrificial bonding behavior, ${ }^{6,7}$ interaction between amorphous and crystalline domains, ${ }^{8,9}$ fraction of order, ${ }^{10}$ and strength of bonding between constituents. ${ }^{11}$ In collagen, size and interactions between helices are similarly tuned for high toughness. ${ }^{2,12}$ At larger scales, 'hard' materials such as bone and nacre (abalone shell) are known to gain toughness through the arrangement and interactions between their soft and hard components: the roughness of the interfaces, ${ }^{13}$ bridging between layers, ${ }^{14,15}$ self-repair mechanisms, and the viscoplastic properties of their soft organic constituents ${ }^{16}$ combine to produce phenomenally strong yet tough materials, far beyond what would be expected from their relatively brittle and weak components. ${ }^{17-20}$ The hierarchical structuring of such materials further decreases their sensitivity to flaws. ${ }^{21}$ Soft tissues such as tendon, ligaments, cartilage, and fascia similarly use combinations of weak component materials to form tough structures. The main component of these materials, collagen, gains considerable strength from its hierarchical structuring, ${ }^{12}$ which is further enhanced by structure at the microscopic to tissue levels. In cartilage, for example, a highly entangled and crosslinked meshwork of collagen entraps highswelling glycosaminoglycan (GAG) to form the incredibly tough tissue on the articulating surfaces of joints, which processes compressive, shear, and tensile stresses through the control of fluid movement. ${ }^{22}$ Tendons and
ABSTRACT Spider silk is a fascinating natural composite material. Its combination of strength and toughness is unrivalled in nature, and as a result, it has gained considerable interest from the medical, physics, and materials communities. Most of this attention has focused on the one to tens of nanometer scale: predominantly the primary (peptide sequences) and secondary ( $\boldsymbol{\beta}$ sheets,

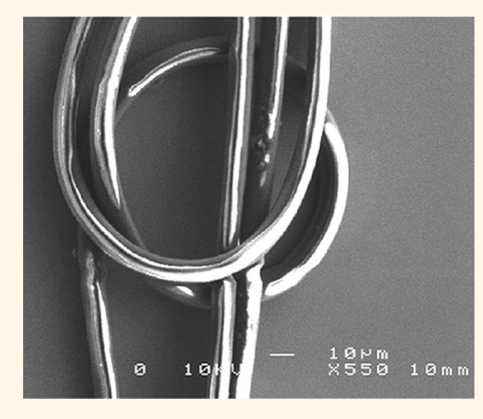
helices, and amorphous domains) structure, with some insights into tertiary structure (the arrangement of these secondary structures) to describe the origins of the mechanical and biological performance. Starting with spider silk, and relating our findings to collagen fibrils, we describe toughening mechanisms at the hundreds of nanometer scale, namely, the fibril morphology and its consequences for mechanical behavior and the dissipation of energy. Under normal conditions, this morphology creates a nonslip fibril kinematics, restricting shearing between fibrils, yet allowing controlled local slipping under high shear stress, dissipating energy without bulk fracturing. This mechanism provides a relatively simple target for biomimicry and, thus, can potentially be used to increase fracture resistance in synthetic materials.

KEYWORDS: spider silk · collagen · mechanics · fibrils · toughness · finite · element $\cdot$ analysis

ligaments achieve high toughness in tension, also using collagen-collagen and collagenGAG interactions over a range of hierarchical levels. ${ }^{23-25}$

Here, we examine the toughening mechanisms operating at the scale of hundreds of nanometers in spider silk using atomic force microscopy (AFM) and finite element simulation, and relate them to the interactions between collagen fibrils in tissues such as tendon, ligament, fascia, and cartilage.

Widely regarded as a 'super fiber', spider silk has received considerable attention as a potential biomaterial $^{26-28}$ (i.e., as a biocompatible material for implanting in human patients) and is an archetypal protein

\section{* Address correspondence to cameron.brown@ndorms.ox.ac.uk, rosei@emt.inrs.ca.}

Received for review July 19, 2011 and accepted February 11, 2012.

Published online February 11, 2012 $10.1021 / \mathrm{nn} 300130 \mathrm{q}$

() 2012 American Chemical Society 

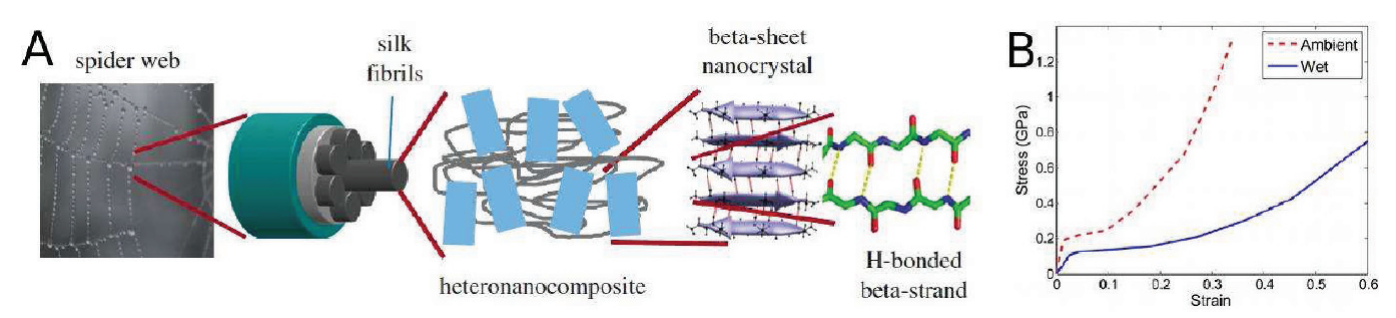

Figure 1. (A) Hierarchical organization of spider silk, reproduced with permission from Keten and Buehler. ${ }^{8}$ Copyright 2010 The Royal Society. (B) Stress-strain behavior of wet and dry spider silk.

elastomer allowing insights into less-accessible biological materials. ${ }^{10}$ A large body of fundamental work on structure-function relationships $s^{7,29}$ and synthesis ${ }^{30,31}$ has also been performed. The bulk mechanical properties are extremely impressive, with an ultimate strength of $1.2 \mathrm{GPa}$ and toughness of $158 \mathrm{~J} \mathrm{~cm}^{-3}$ under tensile load. ${ }^{32}$ As shown in Figure 2, the ability to resist shear stress, in addition to high tensile (outside of bend) and compressive (inside of bend) stresses, is outstanding. There remains, however, a significant gap between our knowledge of the fiber's bulk properties and our understanding of their structural origins, a gap manifested in the relatively poor mechanical performance of synthetic silks which, at best, can withstand approximately one-third the applied stress of natural silks. $^{33,34}$

An important, and so far overlooked, mechanism contributing to the performance of natural silk can be deduced from the fibrils at the core of the fiber. The existence of fibrils in spider silk has been known for some time. ${ }^{35-37}$ The role of fibrils in mechanical models, however, has been largely ignored, and the material treated as having homogeneous properties over its cross section. ${ }^{4,38}$ Although this assumption is valid for axial tension with fibrils aligned parallel to the fiber, and for small strains, our findings suggest that the interaction of fibrils will be important at high strains, particularly in bending, torsion, and combined loading situations which involve high shear stress between fibrils.

\section{RESULTS AND DISCUSSION}

Throughout the core regions of the spider silk fiber, we found $200 \mathrm{~nm}$ diameter fibrils (AFM image, Figure 3) aligned top-left to bottom-right, which is slightly off-axis $\left(\sim 10^{\circ}\right)$ with respect to the overall fiber. The apparent topright to bottom-left alignment shown in Figure $3 \mathrm{~A}$ is along the cutting direction, and we interpret these lines as scratches from an imperfect microtome blade. Collagen images from fascia (Figure $3 \mathrm{E}$ ) and tendon (Figure 3F) show a similar interlocking structure.

The size and orientation of the fibrils observed here are consistent with previous findings, ${ }^{39-41}$ which show braided bundles of fibrils on the hundreds of nanometer scale constituting the fiber's core. Building on the observations from these studies, we found that the

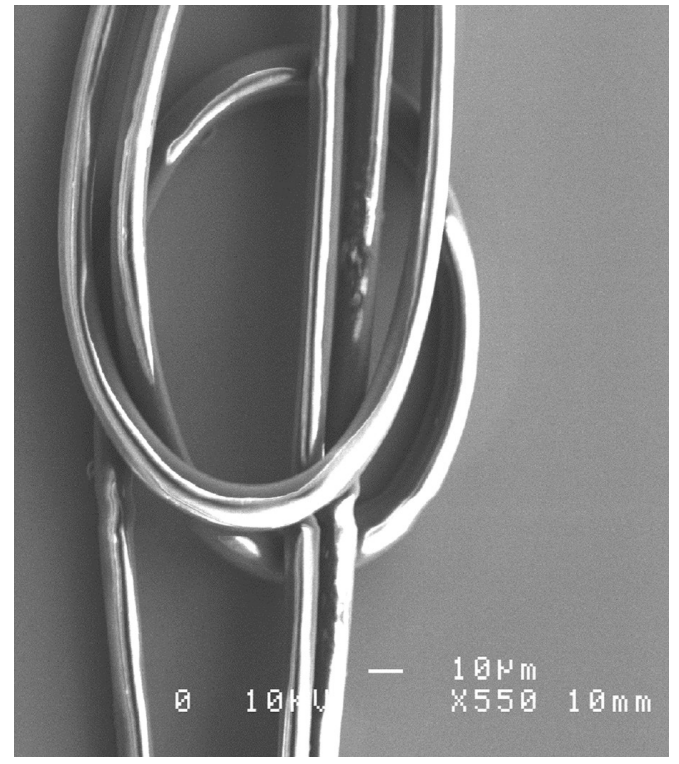

Figure 2. SEM of a knot of spider silk, illustrating impressive ductility and toughness under shear, and the ability to withstand both compressive and tensile stresses. This shows an absence of damage to the inside regions of the bends, which are under large compressive stress, or the outer regions of the bend, which are under large tensile stress.

fibrils are not smooth, but rather characterized by globular protrusions approximately $100 \mathrm{~nm}$ wide and $150 \mathrm{~nm}$ long with respect to the fibril direction (Figure 3B-D). Small cracks/voids were observed in the images; however, these imperfections may be due to the cutting protocol rather than intrinsic structural features. Dense arrays of collagen have a similar interlocking pattern (Figure 3E,F).

2-D structures for finite element simulation were built based on the 'resting' morphology of the spider silk and collagen structures before loading was applied, and the structures allowed to undergo contact and large deformation to simulate the behavior under loading. The simulations showed that the globular/ banding morphology affects the interaction behavior of the fibrils and may therefore affect the mechanical behavior of the core material as a whole by providing a mechanism that combines high stiffness and strength with high toughness.

At low applied shear forces in spider silk (Figure 4), the fibrils remained locked together and the applied 


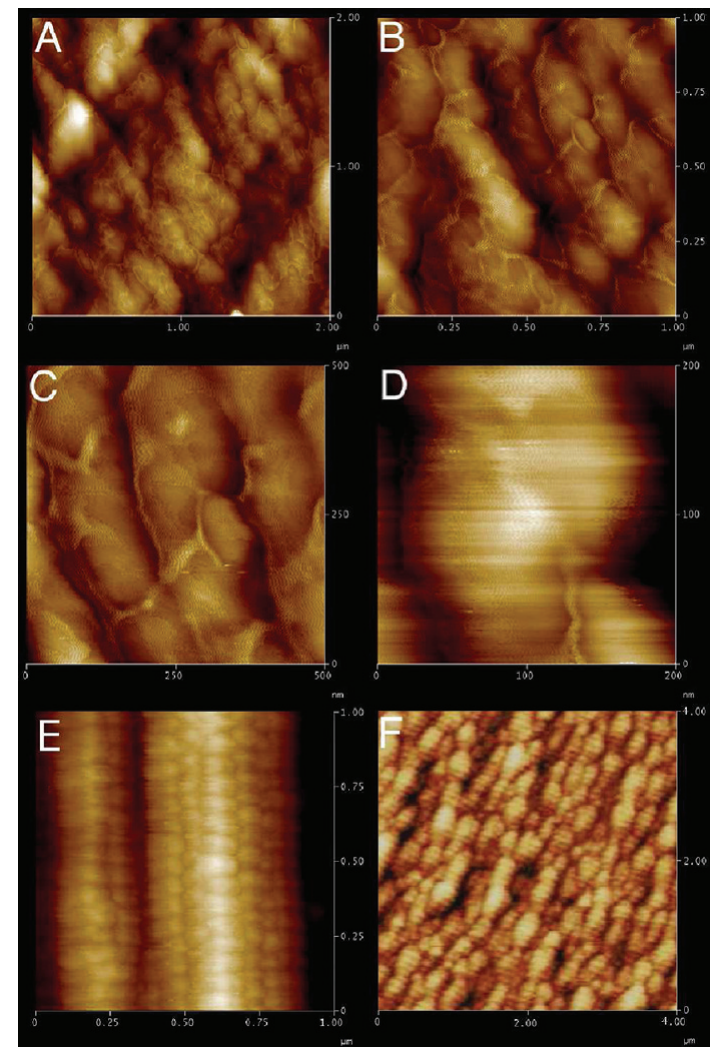

Figure 3. AFM image of fibril structure across a range of size scales. (A-D) Fibrils in the core region of spider silk fibres; (E) two bundles of interlocking collagen fibrils in fascia; (F) collagen in tendon. The scales of panels $A, B, E$, and $F$ are in micrometers; panels $C$ and $D$ are in nanometers. The clear globular/banding patterns can be seen in each fibril and the interlocking of the globules/bands between the fibrils. The authors thank J. Tilley for providing the image in panel $\mathrm{F}$.

shear was carried through the contact between globules, with contact stress increasing sharply with increasing applied shear (Figure 5A). This correlates with the linear stress-strain behavior at the early stages of deformation $^{32}$ and the immediate transfer of stress to the crystalline structure. ${ }^{42}$ With sufficient applied shear, the fibrils slip, dissipating energy through friction (Figures 4 and 5). Because of their braided arrangement, ${ }^{39,40}$ an applied axial stress will produce shear stress between the fibrils, which will be magnified by bending and torsional loads, so that the fibrils try to slide past each other. For the $10^{\circ}$ off-axis alignment of the fibrils observed here, up to one-sixth of the applied bulk axial stress (based on Mohr's circle ${ }^{43}$ ) that is carried by the inner core material will be experienced as shear between the fibrils.

Extrapolating our plane strain simulation to the bulk fiber mechanics by adjusting out of plane contact distance and scaling of applied shear loads to the bulk case indicates that slipping occurs at approximately $550 \mathrm{MPa}$ of applied axial stress, that is, during strain hardening in the axial case. For a combined loading condition, including axial loads, torsional loads, and/or local bending, which may occur due to insect strike in the natural application, bullet strike in the widely proposed bullet proof vest application, or weaving/ braiding/tying in medical and other industrial application, the internal shear will be higher and less uniform, invoking the slipping mechanism under lower applied loads.

A number of factors affect the balance of forces (see Figure 6) and the critical point at which slipping occurs. The sliding condition can be simplified and represented at a point to determine the sensitivity to different geometrical and loading conditions. If the compressive force applied between the fibrils (blue arrows) is $F_{C}$, the shearing force between the fibrils (red arrows) is $F_{\mathrm{S}}$ and the slope of the contact faces is $\theta$, the friction force $F_{\mathrm{fr}}$ required to prevent sliding (black arrow along contact face) is $F_{\mathrm{fr}}=F_{\mathrm{C}} \sin \theta-F_{\mathrm{S}} \cos \theta$, based on the tangential components of the applied loads. This friction force can be calculated as $F_{\mathrm{fr}}=\mu F_{\mathrm{N}}=$ $\mu\left(F_{\mathrm{C}} \cos \theta+F_{\mathrm{S}} \sin \theta\right)$, where $F_{\mathrm{N}}$ is the normal reaction force (large black arrow) and $\mu$ is the effective coefficient of friction. This provides a critical sliding requirement of $F_{\mathrm{C}} \sin \theta-F_{\mathrm{S}} \cos \theta>\mu\left(F_{\mathrm{C}} \cos \theta+F_{\mathrm{S}} \sin \theta\right)$.

On the basis of the simplification in Figure 6, compressive forces between the fibrils (depend on packing density and the type of load applied) act to resist sliding with the component of compressive force at the contact angle and friction through the component normal to the contact. The fibril morphology will determine the angle of the contact region and, therefore, the components of compression and reaction forces acting to resist sliding.

This behavior, however, is complicated by the stiffness of the fibril material and the effective friction coefficient between adjacent fibrils. The former (Figure 7) determines the contact area and therefore the total friction (soft fibrils deform at contact, increasing the contact area). Coupled with increasing the contact area, the deformation in soft fibrils reduces the contact angle and, therefore, the resistance to sliding, counteracting the increase in the total friction. High stiffness further leads to high contact stress as the load is applied to a small area. The complex interrelationships between the different factors suggest that an optimal material stiffness exists for any given loading situation.

The effective friction coefficient determines the resistance to sliding, yet also the distribution of stress within the globules/bands (Figure 8). For low coefficients of friction (for $\mu<0.3$ ), the maximum stresses occur within the fibril. As friction is increased, the maximum stress occurs gradually closer to the interface as the contact plays an increasing role in resistance to sliding $(\mu>0.3)$ and the critical shear load required for sliding increases. At high coefficients of friction (above $\mu=0.8$ for this configuration), the high stresses at the interface may damage the structure, further indicating an optimal material property specification. 

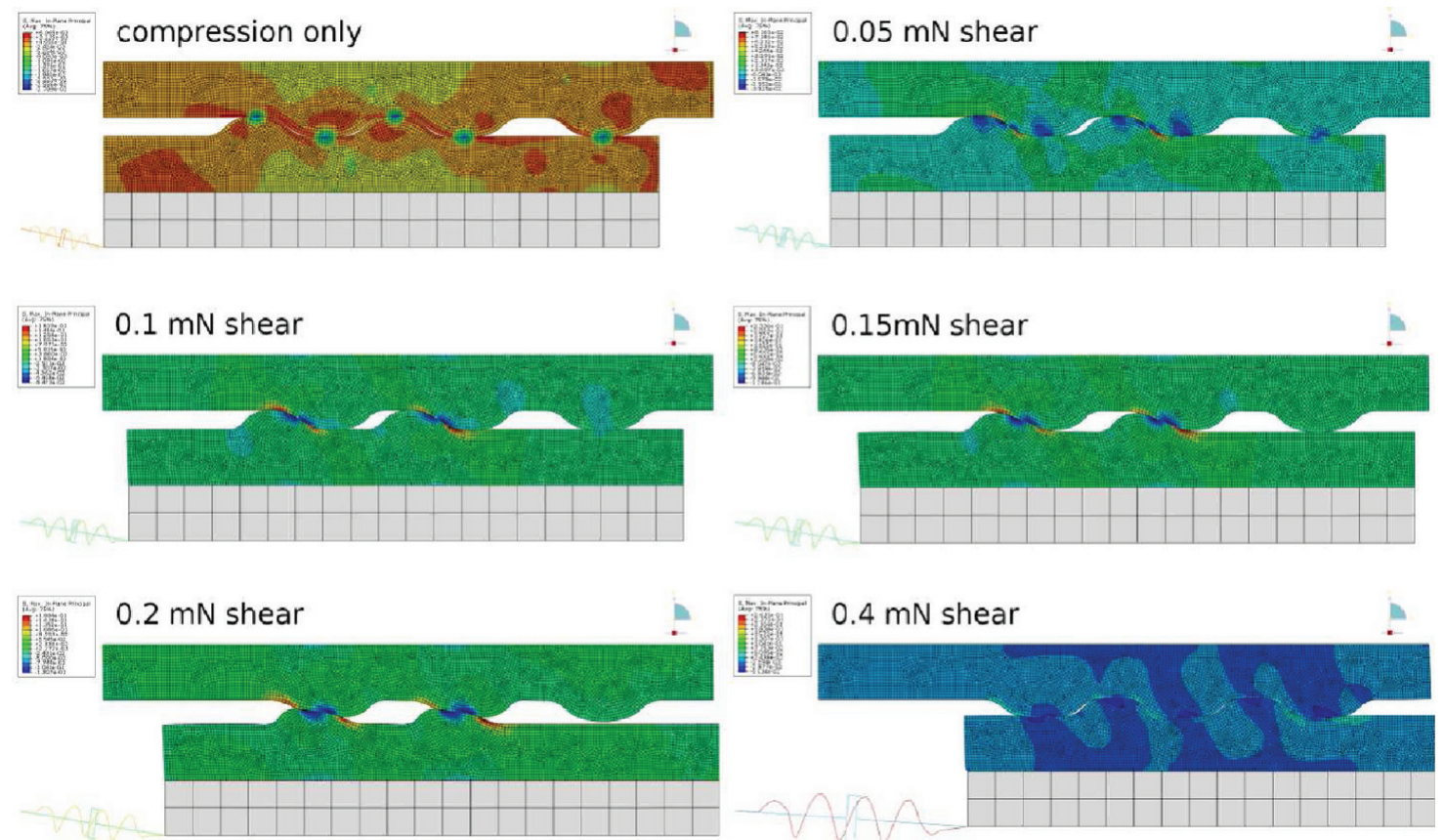

Figure 4. Finite element simulation of fibril interaction, showing deformation and the maximum principal stress. Shear is applied from left to right on the lower fibril. At rest and under low loads, the fibrils are locked together by the globules but, under sufficient shear stress, can 'slip-and-hold', sliding past each other in 100-150 nm steps, corresponding to the longitudinal ordering of the globules. Slipping occurs at approximately $0.2 \mathrm{mN}$.
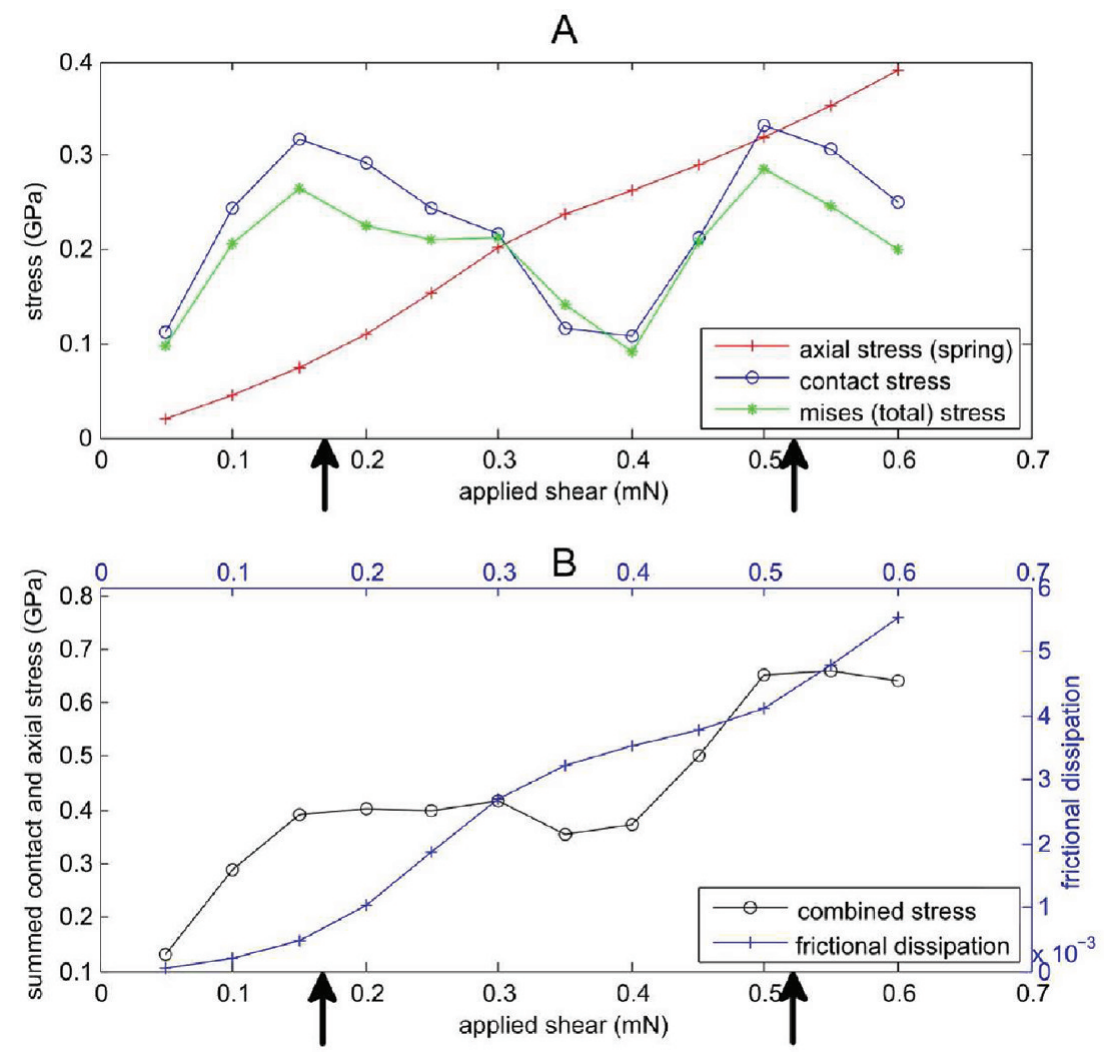

Figure 5. Sharing between contact and axial stresses under different applied shear loads, based on a material stiffness of $1 \mathrm{GPa}$ and coefficient of friction of $\mathbf{0 . 5}$. Arrows mark the points at which slipping occurs. (A) The stress carried in contact (principal stress), the maximum total stress, excluding axial stress, and the axial stress represented by the spring; (B) the summed contact and axial stress to illustrate the total stress in the fiber at different stages of slipping, with the cumulative frictional energy dissipation overlaid. Contact stress increases up to $0.15 \mathrm{mN}$ before the restoring and friction forces are overcome and slip occurs. The fibrils relock at $0.4 \mathrm{mN}$. 
Once the balance of forces holding the fibrils in place are overcome and slipping occurs, the fibrils lock into position against the adjacent globule. The combination of axial stiffness and morphology restrict local deformation to $150-200 \mathrm{~nm}$ steps in spider silk or $\sim 70 \mathrm{~nm}$ steps in collagen, corresponding to the longitudinal spacing of globules or bands, respectively. Here, the high contact stresses (Figures 4 and 5) between the fibrils are transferred to bulk axial stress (represented as the spring) upon sliding, and the contact stress at the subsequent lock-in point is of a similar magnitude, despite a 2 -fold increase in applied shear. When the contact and axial stress is combined (Figure 5B), it appears that elastic energy is stored in the material up to each point of sliding. During the sliding step, the total stress carried by the fibrils remains constant despite increasing applied loads, with energy dissipated through friction.

As the fibrils maintain their configuration after sliding, the dislocation is unlikely to cause the stress concentrations that drive crack progression. ${ }^{44}$ On the basis of the similar geometry, the similar contact conditions before and after slipping, the increase in

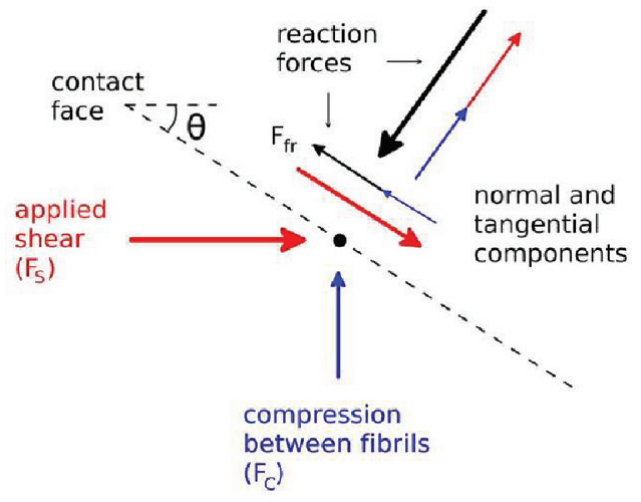

Figure 6. Free body diagram of contact reduced to a point, illustrating the requirement for slipping as a loss of balance in the tangential forces. axial stress between the lock-in points (Figure 5A), and the 'discrete step' pattern of summed stress in the fibrils (Figure 5B), it is unlikely that slipping will continue at the same interface. If the behavior is considered in the context of a tight bundle or network of fibrils, we argue that dislocations will be sequentially spread over a large volume of material due to the highly localized relief of contact stress that accompanies the slip. In this situation, the slip-and-hold behavior provides a toughening mechanism that can occur repeatedly over the large deformations (up to $60 \%$ strain, Figure 1B) experienced by the fiber.

This controlled yet limited nanoscale sliding, spread over a large volume, is similar to that which produces the high toughness of nacre ${ }^{18}$ in which stiff aragonite layers have been shown to slide over one another, aided by the viscoplastic deformation of an organic 'glue' layer. ${ }^{17,45}$ It is unclear, however, whether an intermediary substance acts between spider silk fibrils. Voids between the fibrils may contain unbound water which will act as a lubricant, or as a reservoir to drive recovery of the nanostructure. ${ }^{29}$ Some evidence of glycoprotein has also been shown in the spider silk core, based on Concanavalin A reactivity. ${ }^{40}$ A glycoprotein layer would be expected to act to resist slipping, increasing the effective friction at the interface (see Figure 8). No strong evidence of interfibrillar material was observed in the AFM images; however, the Citrisolv (see Experiments and Simulation) used to remove paraffin may act to remove this substance, if it indeed exists. Further work will be required to identify and mechanically characterize interfibrillar material in the spider silk core, particularly to link the behavior of fibrils in spider silk to those of other biological fibrils/ fibres.

Modification of the geometry (Figure 3 ) and stiffness ${ }^{46}$ to represent collagen shows similar slipping behavior to that of spider silk (see Figure 9), suggesting that the controlled sliding mechanism may be present in a

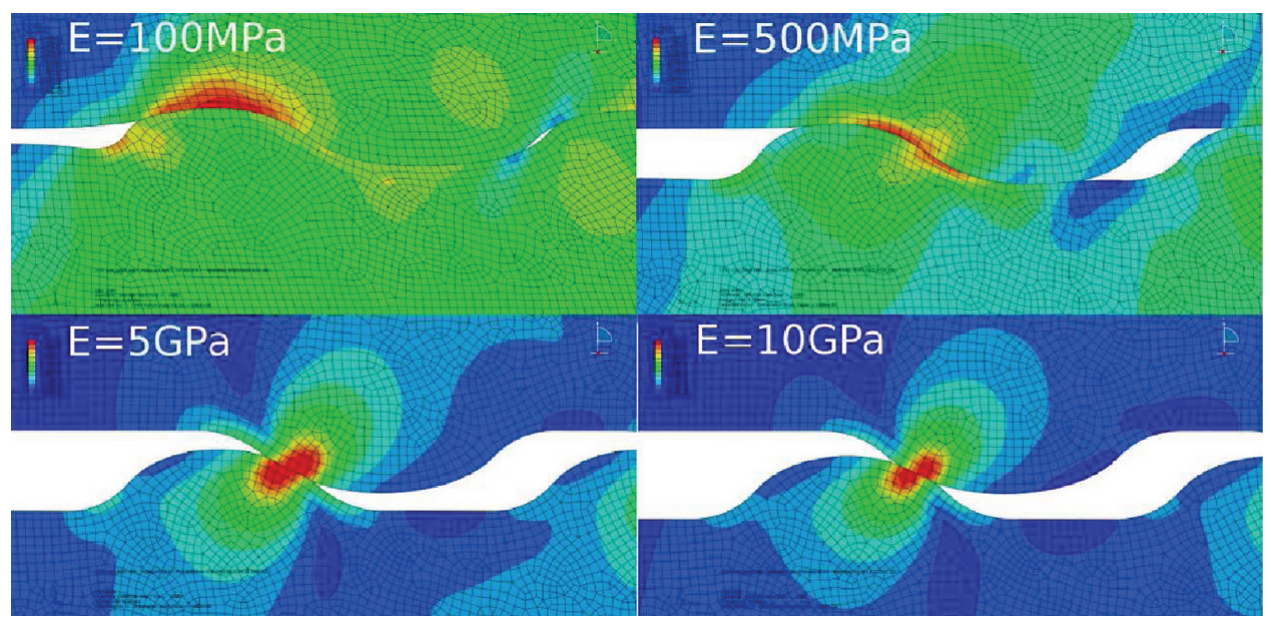

Figure 7. FE simulation showing (with von Mises stress distribution) the effect of stiffness on fibril behavior under $0.1 \mathrm{mN}$ of applied shear (left to right on the lower fibril). High stiffness maintains a high contact angle, yet reduces the contact area. 


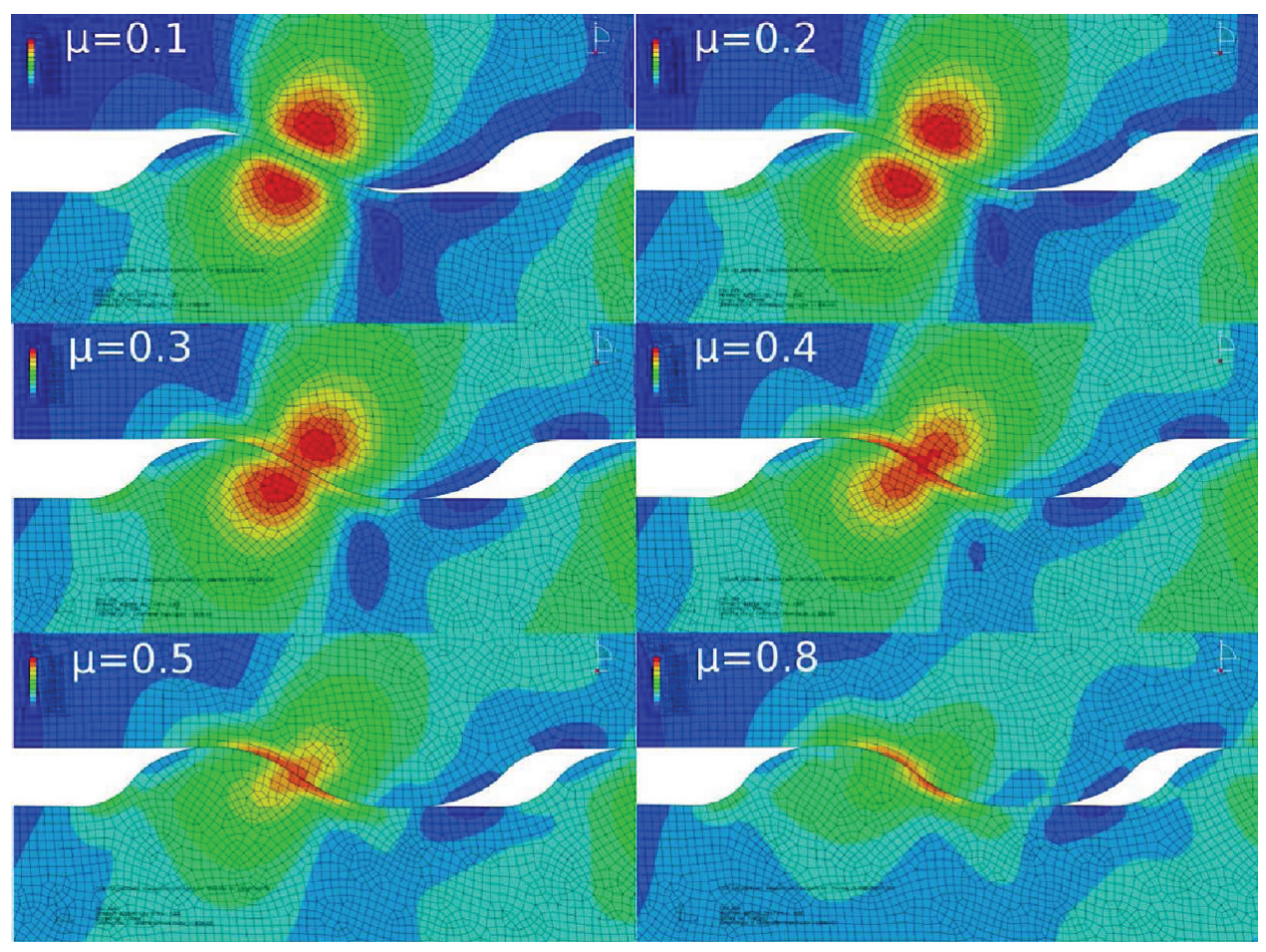

Figure 8. FE simulation showing (with von Mises stress distribution) the effect of the coefficient of friction under $0.1 \mathrm{mN}$ of applied shear (left to right on the lower fibril). High coefficients of friction cause high stresses at the contact surface. This increases the resistance to sliding, yet the high stress may lead to damage of the material.
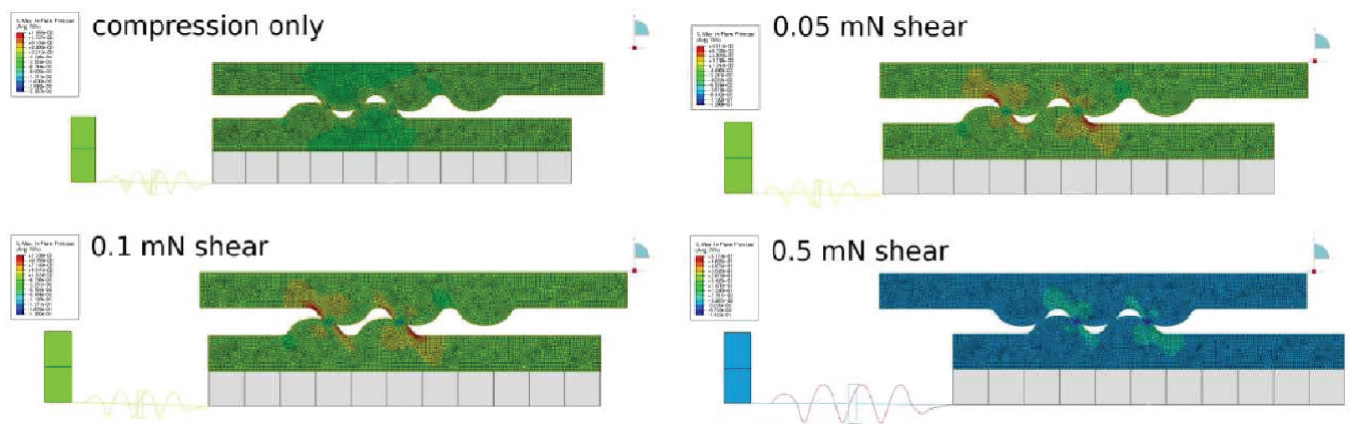

Figure 9. Finite element simulation of collagen fibril interaction, showing deformation and the maximum principal stress. Here, slipping occurs in approximately $70 \mathrm{~nm}$ steps, corresponding to the banding pattern.

number of collagenous tissues. The collagen simulation does not, however, share stress effectively between axial and contact stresses, with contact stress increasing gradually with increasing shear rather than decreasing during sliding as in spider silk. Increasing the resistance to axial deformation restores this balance, suggesting an increased role of interfibril material or cross-linking in collagenous structures. Although collagen-collagen interaction is arguably dominated by this cross-linking in sparse matrices (e.g., deep zone cartilage), sliding will be important where collagen is dense and aligned. Such tissues include fascia, tendons, ligaments, and some regions of cartilage (surface zone and the chondron sheath). At $8 \%$ strain in tendon, for example, less than $4 \%$ strain is experienced by the fibrils, which is thought to occur due to sliding effects, ${ }^{24,47}$ combined with the deformation of
GAG. Controlled slipping has also been observed in active processes such as the sliding of actin and myosin filaments ( $2.7 \mathrm{~nm}$ steps) in muscle activity. ${ }^{48}$

The controlled sliding between fibrils and the spread of local dislocations over a large volume of material that result from this fibril morphology could potentially provide a toughening mechanism in synthetic materials. Controlled local fracture has already been successfully attempted in hard materials (e.g., Al2O3/PMMA composites), ${ }^{18}$ using a 'brick and mortar' structure to increase fracture resistance at low strains. We believe that the repeated, interlocking globular morphology of spider silk fibrils may be replicated in fibrous materials to combine high strength and stiffness with high toughness, in terms of energy to failure, which will act at high strains. Because of the larger scale (hundreds 
of nanometers) features on which this energy dissipating mechanism acts, control over this morphology will be a relatively simple target for biomimicry. Rather than relying on the basic material properties of a fiber or fibril, it requires only morphological manipulation or design. On the micrometer scale, such morphological adaptations can be achieved through the modification of spinnerets in fiber spinning setups to include a threading or ribbing stage, effectively by forging, as the fibers are drying or precipitating. The size, shape, and distance between beads in electrospun nanofibers can be controlled by polymer concentration, solvent used, and applied voltage. ${ }^{49}$ More subtle surface roughening has also been shown in electrospun fibers through the control of basic processing parameters, ${ }^{50}$ and surface morphology can be further manipulated through postprocessing such as chemical etching, plasma, and ion beam treatments. Such modifications may have real potential for industrial application in cables or fiber-reinforced composites, or in the production of tissue engineered constructs seeking to replicate tendons and ligaments.

\section{CONCLUSIONS AND PERSPECTIVES}

We have described toughening mechanisms occurring at the hundreds of nanometer scale in spider silk and collagen-dense tissues using AFM combined with finite element simulation. The globular fibril morphology in spider silk, and the regular banding in collagen, locks the fibrils together, restricting shearing between fibrils, yet allows controlled local slipping under high shear stress. The local slipping dissipates energy without bulk fracturing. This mechanism provides a relatively simple target for biomimicry and, thus, can potentially be used to increase fracture resistance in synthetic materials.

\section{EXPERIMENTS AND SIMULATION}

Spider silk was harvested from the structural spans of Nephila clavipes (golden orb weaver) web in Redland Bay, Australia, the morning after spinning, with original fiber tension maintained by fixing the ends. The fiber bundles, containing major ampullate silk, were mounted in paraffin, cut into $5 \mu \mathrm{m}$ sections by microtome (RM2155, Leica Mikrosysteme GmbH, Austria), and mounted on a platinum plated silicon wafer. The paraffin was then removed by a sequence of 3 dips in Citrisolv (Fisher Scientific, Ottawa, Ontario, Canada) followed by a Citrisolv/ethanol mixture and a gradual rehydration in a series of distilled water/ethanol mixtures. The samples were left to equilibrate overnight at approximately $60 \%$ relative humidity and $24^{\circ} \mathrm{C}$, leaving an array of samples. The ability to remove paraffin from the samples after sectioning provides a significant advantage over other standard cutting media such as Epon and LR-white, ${ }^{40}$ which penetrate the material and harden, thus, altering its mechanical properties and 'smoothing' the AFM image. The sections were imaged in tapping mode under ambient conditions using an atomic force microscope (Enviroscope, Veeco Instruments, Inc., Santa Barbara, CA), with a tip of nominal radius of $10 \mathrm{~nm}$ (T300, VISTAprobes, Phoenix, AZ). The cantilever had a resonant frequency of $292 \mathrm{kHz}$.

An explicit plane strain finite element simulation was generated in ABAQUS (version 6.8.4, Simula Corporation, Providence, $\mathrm{RI})$ to analyze the mechanical effects of the fibril morphology determined from AFM imaging. Finite element simulation has been successfully applied at this scale in the study of plate sliding in nacre and bone mechanics. ${ }^{17,45}$ The explicit finite element approach was chosen here as an efficient method for simulating large deformation and contact, and for the ease of comparison between fibrils due to precise control over geometry, interfibril friction, and material stiffness within the assumption of a continuous material. This continuum assumption may, however, present a limitation of our approach compared to molecular dynamics $^{51}$ and coarse graining, ${ }^{52}$ which take into account the complex structural response to loading on the $1-100 \mathrm{~nm}$ scale. The present model does not attempt to represent the complex deformation within the fibril, restructuring within the fibril under axial tension, or the presence of residual stress but rather assumes a simplified distribution of stress to simulate contact and sliding on the hundreds of nanometer scale.

The $200 \mathrm{~nm}$ diameter fibril sections with $140 \times 40 \mathrm{~nm}$ globule protrusions were assigned linear elastic properties with an initial elastic modulus of $1 \mathrm{GPa}$ based on the bulk elastic modulus of hydrated silk. ${ }^{10}$ A Poisson's ratio of 0.35 was used to account for the compressibility of the disordered domains and restructuring under load. ${ }^{53}$ Stiffness was varied to represent the elastic modulus of bulk silk in ambient conditions (10 GPa), ${ }^{10}$ and repeated at $100 \mathrm{MPa}, 500 \mathrm{MPa}$, and $100 \mathrm{GPa}$ for comparison. The upper surface of the upper fibril was fixed in space. The lower fibril was bonded to a rigid block to which an arbitrary distributed load of $5 \mathrm{MPa}$ was applied to provide firm contact between the fibrils. A stepwise increasing force of $0.05 \mathrm{mN}$ every $2 \mathrm{~s}$ was applied for $32 \mathrm{~s}$ to simulate shear between the fibrils. A damped spring was attached to the lower fibril to simulate axial tension. Four-node bilinear quadrilateral elements (CPE4R) were used, with a mesh size of $5 \mathrm{~nm}$. To check mesh size, a simulation was run using a mesh size of $1 \mathrm{~nm}$. Overall behavior remained unchanged, and von Mises stress ${ }^{54}$ was similar.

Surface to surface contact was initially modeled using an effective coefficient of friction of 0.5 . The coefficient was varied from 0 to 0.8 to determine its effect on behavior and to provide insight into the effect of interfibril material acting to facilitate or restrict tangential motion at the contact surfaces. Friction coefficients above 0.8 caused material failure due to high contact stresses. The effective coefficient of friction in our model derives from a classical definition of friction, dependent on contact area and normal force which, at this scale, includes van der Waals forces of the order of a few nanonewtons $(\mathrm{nN})$ per contact point. The model does not explicitly include influence from an intermediary material between the contact surfaces.

Conflict of Interest: The authors declare no competing financial interest

Acknowledgment. This work was supported by grants from The Ministry for Foreign Affairs of Italy (Direzione Generale per la Promozione e la Cooperazione Culturale) and MDEIE (Québec) under the framework of the Italy-Québec Joint Laboratory in Nanostructured Materials for Energy, Catalysis and Biomedical Applications. C.P.B. acknowledges the Fonds de la Recherche en Santé Québec, Canada for a personal fellowship. F.R. is grateful to the Canada Research Chairs program for partial salary support. C.H. acknowledges Natural Sciences and Engineering Research Council of Canada for financial support. Financial support has been received from the NIHR Biomedical Research Unit into Musculoskeletal Disease, Nuffield Orthopaedic Centre and University of Oxford, and Arthritis Research UK.

\section{REFERENCES AND NOTES}

1. Omenetto, F. G.; Kaplan, D. L. New Opportunities for an Ancient Material. Science 2010, 329, 528-531. 
2. Weinkamer, R.; Fratzl, P. Nature's Hierarchical Materials. Prog. Mater. Sci. 2007, 52, 12631334.

3. Keten, S.; Buehler, M. J. Geometric Confinement Governs the Rupture Strength of $\mathrm{H}$-Bond Assemblies at a Critical Length Scale. Nano Lett. 2008, 8, 743-748.

4. Termonia, Y. Molecular Modelling of Spider Silk Elasticity. Macromolecules 1994, 27, 7378-7381.

5. Keten, S.; Xu, Z.; Ihle, B.; Buehler, M. J. Nanoconfinement Controls Stiffness, Strength and Mechanical Toughness of Beta-Sheet Crystals in Silk. Nat. Mater. 2010, 9, 359-367.

6. Fantner, G. E.; Oroudjev, E.; Schitter, G.; Golde, L. S.; Thurner, P.; Finch, M. M.; Turner, P.; Gutsmann, T.; Morse, D. E.; Hansma, H.; Hansma, P. K. Sacrificial Bonds and Hidden Length: Unraveling Molecular Mesostructures in Tough Materials. Biophys. J. 2006, 90, 1411-1418.

7. Brown, C. P.; MacLeod, J.; Amenitsch, H.; Cacho-Nerin, F.; Gill, H. S.; Price, A. J.; Traversa, E.; Licoccia, S.; Rosei, F. The Critical Role of Water in Spider Silk and Its Consequence for Protein Mechanics. Nanoscale 2011, 3, 3805-3811.

8. Keten, S.; Buehler, M. J. Nanostructure and Molecular Mechanics of Dragline Spider Silk Protein Assemblies. J. R. Soc. Interface 2010, 7, 1709-1721.

9. Nova, A.; Keten, S.; Pugno, N. M.; Redaelli, A.; Buehler, M. J. Molecular and Nanostructural Mechanisms of Deformation, Strength and Toughness of Spider Silk Fibrils. Nano Lett. 2010, 10, 2626-2634.

10. Vollrath, F.; Porter, D. Spider Silk as Archetypal Protein Elastomer. Soft Matter 2006, 2, 377-385.

11. Lee, S. M.; Pippel, E.; Gösele, U.; Dresbach, C.; Qin, Y.; Chandran, C. V.; Bräuniger, T.; Hause, G.; Knez, M. Greatly Increased Toughness of Infiltrated Spider Silk. Science 2009, 324, 488-492.

12. Gautieri, A.; Vesentini, S.; Redaelli, A.; Buehler, M. J. Hierarchical Structure and Nanomechanics of Collagen Microfibrils from the Atomistic Scale Up. Nano Lett. 2011, 11, 757-766.

13. Song, F.; Soh, A. K.; Bai, Y. L. Structural and Mechanical Properties of the Organic Matrix Layers of Nacre. Biomaterials 2003, 24, 3623-3631.

14. Foulk, J. W.; Johnson, G. C.; Klein, P. A.; Ritchie, R. O. On the Toughening of Brittle Materials by Grain Bridging: Promoting Intergranular Fracture through Grain Angle, Strength, and Toughness. J. Mech. Phys. Solids 2008, 56, 2381-2400.

15. Nalla, R. K.; Stolken, J. S.; Kinney, J. H.; Ritchie, R. O. Fracture in Human Cortical Bone: Local Fracture Criteria and Toughening Mechanisms. J. Biomech. 2005, 38, 15171525.

16. Smith, B. L.; Schäffer, T. E.; Viani, M.; Thompson, J. B.; Frederick, N. A.; Kindt, J.; Belcher, A.; Stucky, G. D.; Morse, D. E.; Hansma, P. K. Molecular Mechanistic Origin of the Toughness of Natural Adhesives, Fibres and Composites. Nature 1999, 399, 761-763.

17. Barthelat, F.; Rabiei, R. Toughness Amplification in Natural Composites. J. Mech. Phys. Solids 2011, 59, 829-840.

18. Munch, E.; Launey, M. E.; Alsem, D. H.; Saiz, E.; Tomsia, A. P.; Ritchie, R. O. Tough, Bio-Inspired Hybrid Materials. Science 2008, 322, 1516-1520.

19. Mayer, G. Rigid Biological Systems as Models for Synthetic Composites. Science 2005, 310, 1144-1147.

20. Sen, D.; Buehler, M. J. Structural Hierarchies Define Toughness and Defect-Tolerance Despite Simple and Mechanically Inferior Brittle Building Blocks. Sci. Rep. 2011, 1 No. Article 35.

21. Gao, H.; Baohua, J.; Jäger, I. L.; Arzt, E.; Fratzl, P. Materials Become Insensitive to Flaws at Nanoscale: Lessons from Nature. Proc. Natl. Acad. Sci. U.S.A. 2003, 100, 5597-5600.

22. Mow, V. C.; Kuei, S. C.; Lai, M. W.; Armstrong, C. G. Biphasic Creep and Stress Relaxation of Articular Cartilage in Compression: Theory and Experiments. J. Biomech. Eng. 1980, 102, 73-84.

23. Lakes, R. Materials with Structural Hierarchy. Nature 1993, 361, 511-515.

24. Puxkandl, R.; Zizak, I.; Paris, O.; Keckes, J.; Tesch, W.; Bernstorff, S.; Purslow, P.; Fratzl, P. Viscoelastic Properties of Collagen: Synchrotron Radiation Investigations and
Structural Model. Philos. Trans. R. Soc. London, Ser. B 2002, 357, 191-197.

25. Tilley, J.; Carr, A. J.; Czernuszka, J. T. Atomic Force Microscopy of Bulk Tendon Samples: Affect of Location and Fixation on Tissue Ultrastructure. Micron 2011, 42, 531535.

26. Brown, C. P.; Rosei, F.; Traversa, E.; Licoccia, S. Spider Silk as a Load-Bearing Biomaterial: Tailoring Mechanical Properties Via Structural Modifications. Nanoscale 2011, 3, 870876.

27. Altman, D. G.; Diaz, F.; Jakuba, C.; Calabro, T.; Horan, R. L.; Chen, J.; Lu, H. H.; Richmond, J.; Kaplan, D. L. Silk-Based Biomaterials. Biomaterials 2003, 24, 401-416.

28. Vepari, C.; Kaplan, D. L. Silk as a Biomaterial. Prog. Polym. Sci. 2007, 32, 991-1007.

29. Liu, Y.; Shao, Z.; Vollrath, F. Relationships between Supercontraction and Mechanical Properties of Spider Silk. Nat. Mater. 2005, 4, 901-905.

30. Holland, C.; Terry, A. E.; Porter, D.; Vollrath, F. Comparing the Rheology of Native Spider and Silkworm Spinning Dope. Nat. Mater. 2006, 5, 870-874.

31. Vollrath, F.; Knight, D. P. Liquid Crystalline Spinning of Spider Silk. Nature 2001, 410, 541-548.

32. Denny, M. The Physical Properties of Spider's Silk and Their Role in the Design of Orb-Webs. J. Exp. Biol. 1976, 65, 483506.

33. Vollrath, F.; Porter, D.; Holland, C. There Are Many More Lessons Still to Be Learned from Spider Silks. Soft Matter 2011, 7, 9595-9600.

34. Holland, C.; Terry, A. E.; Porter, D.; Vollrath, F. Natural and Unnatural Silks. Polymer 2007, 48, 3388-3392.

35. Augsten, K.; Muhlig, P.; Herrmann, C. Glycoproteins and Skin-Core Structure in Nephila Clavipes Spider Silk Observed by Light and Electron Microscopy. Scanning 2000, 22, 12-15.

36. Li, S. F. Y.; McGhie, A. J.; Tang, S. L. New Internal Structure of Spider Dragline Silk Revealed by Atomic Force Microscopy. Biophys. J. 1994, 66, 1209-1212.

37. Vollrath, F.; Holtet, T.; Thogersen, H. C.; Frische, S. Structural Organization of Spider Silk. Proc. R. Soc. London, Ser. B 1996, 263, 147-151.

38. Porter, D.; Vollrath, F.; Shao, Z. Predicting the Mechanical Properties of Spider Silk as a Model Nanostructured Polymer. Eur. Phys. J. E: Soft Matter Biol. Phys. 2005, 16, 199206.

39. Gould, S. A. C.; Tran, K. T.; Spagna, J. C.; Moore, A. M. F.; Shulman, J. B. Short and Long Range Order of the Morphology of Silk from Latrodectus Hesperus (Black Widow) as Characterized by Atomic Force Microscopy. Int. J. Biol. Macromol. 1999, 24, 151-157.

40. Sponner, A.; Vater, W.; Monajembashi, S.; Unger, E.; Grosse, F.; Weisshart, K. Composition and Hierarchical Organisation of a Spider Silk. PLoS One 2007, 2, e998.

41. Hakimi, O.; Knight, D. P.; Knight, M. M.; Grahn, M. F.; Vadgama, P. Ultrastructure of Insect and Spider Cocoon Silks. Biomacromolecules 2006, 7, 2901-2908.

42. Papadopoulos, P.; Solter, J.; Kremer, F. Hierarchies in the Structural Organization of Spider Silk-a Quantitative Model. Colloid Polym. Sci. 2009, 287, 231-236.

43. Mohr, O. Uber Die Darstellung Des Spannungszustandes Und Des Deformationszustandes Eines Korper-Elements. Zivil Ingenieur 1882, 28, 113-156.

44. Ritchie, R. O. Mechanisms of Fatigue-Crack Propagation in Ductile and Brittle Solids. Int. J. Fract. 1999, 100, 55-83.

45. Barthelat, F.; Tang, H.; Zavattieri, P. D.; Li, C.-M.; Espinosa, H. D. On the Mechanics of Mother-of-Pearl: A Key Feature in the Material Hierarchical Structure. J. Mech. Phys. Solids 2007, 55, 306-337.

46. Wenger, M. P. E; Bozec, L.; Horton, M. A.; Mesquida, P. Mechanical Properties of Collagen Fibrils. Biophys. J. 2007, 93, 1255-1263.

47. Screen, H. R. C.; Lee, D. A.; Bader, D. L.; Shelton, J. C. An Investigation into the Effects of the Heirarchical Structure of Tendon Fascicles on Micromechanical Properties. Proc. Inst. Mech. Eng., Part H 2004, 218, 109-119. 
48. Liu, X.; Pollack, G. H. Stepwise Sliding of Single Actin and Myosin Filaments. Biophys. J. 2004, 86, 353-358.

49. Tomczak, N.; van Hulst, N. F.; Vancso, G. J. Beaded Electrospun Fibers for Photonic Applications. Macromolecules 2005, 38, 7863-7866.

50. Megelski, S.; Stephens, J. S.; Chase, D. B.; Rabolt, J. F. Microand Nanostructured Surface Morphology on Electrospun Polymer Fibers. Macromolecules 2002, 35, 8456-8466.

51. Keten, S.; Buehler, M. J. Atomistic Model of the Spider Silk Nanostructure. Appl. Phys. Lett. 2010, 96, \#153701.

52. Knowles, T. P.; Fitzpatrick, A. W.; Meehan, S.; Mott, H. R.; Vendruscolo, M.; Dobson, C. M.; Welland, M. E. Role of Intermolecular Forces in Defining Material Properties of Protein Nanofibrils. Science 2007, 318, 1900-1903.

53. Gliovi, A.; Vehoff, T.; Davies, R. J.; Salditt, T. Strain Dependent Structural Changes of Spider Dragline Silk. Macromolecules 2008, 41, 390-398.

54. Mises, R. The Mechanics of Solids in the Plastically-Deformable State. Nachr. Ges. Wiss. Goettingen 1913, 582-592. 